INPLASY

PROTOCOL

To cite: He et al. Efficacy and safety of corticosteroids prophylaxis in cardiac surgery: a protocol for systematic review and meta analysis. Inplasy protocol 2020100044. doi:

10.37766/inplasy2020.10.0044

Received: 13 October 2020

Published: 13 October 2020

Corresponding author: Liang Wan Chen

chenliangwan@fjmu.edu.cn

Author Affiliation:

Fujian Medical University

Union Hospital

Support: 2018YZ0001-1.

Review Stage at time of this submission: Data analysis.

Conflicts of interest:

The authors have no conflicts of interest to disclose.

\section{Efficacy and safety of corticosteroids prophylaxis in cardiac surgery: a protocol for systematic review and meta analysis}

He, J1; Zhang, Y2; Qiu, Z3; Chai, T4; Fang, G5; Hu, Y6; Xu, F7; Huang, Q8; Zheng, $\mathrm{H}^{9}$; Zhou, $\mathrm{H}^{10}$; Tian, $\mathrm{M}^{11}$; Chen, LW12.

Review question / Objective: Although corticosteroid prophylaxis in adult cardiac surgery has been studied extensively for 40 years, its role remains controversial, and the optimal dose remains uncertain. The objective of this meta-analysis was to estimate the clinical benefits and risks of corticosteroid use in cardiopulmonary bypass.

Condition being studied: This study will compare the efficacy of tprophylactic corticosteroids for adults and children undergoing cardiac surgery with CPB. Due to the nature of the disease and intervention methods, randomized controlled trials may be inadequate, and we will carefully consider inclusion in high-quality, non-randomized controlled trials, but this may result in high heterogeneity and affect the reliability of the results.

INPLASY registration number: This protocol was registered with the International Platform of Registered Systematic Review and Meta-Analysis Protocols (INPLASY) on 13 October 2020 and was last updated on 13 October 2020 (registration number INPLASY2020100044).

\section{INTRODUCTION}

Review question / Objective: Although corticosteroid prophylaxis in adult cardiac surgery has been studied extensively for $\mathbf{4 0}$ years, its role remains controversial, and the optimal dose remains uncertain. The objective of this meta-analysis was to estimate the clinical benefits and risks of corticosteroid use in cardiopulmonary bypass.

Rationale: Most cardiac operations are performed under cardiopulmonary bypass, however, it is well known that cardiopulmonary bypass often causes 
systemic inflammatory response syndrome (SIRS). SIRS related to complement, platelets, neutrophils, monocytes, macrophages activation and cascade (coagulation, fibrinolytic, stimulating peptide enzyme), leading to endothelial permeability increase, blood vessels and organ parenchyma cell injury, and liver, kidney, nervous system dysfunction, myocardium injury and infarction, respiratory failure, multiple organ dysfunction, and death are closely related. Corticosteroids is a low-cost drug that can effectively inhibit inflammation, limit systemic capillary leakage syndrome and reduce organ damage, thus providing a theoretical basis for its clinical application. However, corticosteroids may have their own side effects, causing hyperglycemia, which is associated with immunosuppression and poor wound healing. In addition, high doses of corticosteroids were associated with an increased risk of gastrointestinal bleeding and myocardial infarction. The beneficial effects of glucocorticoids on adults and children undergoing heart surgery remain controversial. Three meta-analysis of small RCTs showed that prophylactic corticosteroids can reduce the risk of atrial fibrillation after cardiac surgery in adults, reduce the duration of mechanical ventilation and hospital stay, but can cause some potential side effects. None of the three studies analyzed pediatric studies, and there was a lack of high-quality, largesample randomized controlled trials. The clinical results of the analysis were not comprehensive and the evidence obtained was not robust. Subsequently, two large multicenter randomized controlled trials showed that corticosteroid treatment had no benefit in adult patients undergoing heart surgery and increased the risk of myocardial infarction. However, guidelines for adult cardiac surgery do not recommend routine prophylactic use of corticosteroids to reduce complications. Therefore, the purpose of this study was to systematically review and meta-analyze the dose-dependent benefits and risks of prophylactic glucocorticoids in adults and children undergoing cardiopulmonary bypass.
Condition being studied: This study will compare the efficacy of prophylactic corticosteroids for adults and children undergoing cardiac surgery with CPB. Due to the nature of the disease and intervention methods, randomized controlled trials may be inadequate, and we will carefully consider inclusion in highquality, non-randomized controlled trials, but this may result in high heterogeneity and affect the reliability of the results.

\section{METHODS}

Search strategy: The subject terms and keywords corresponding to Medical Subject Heading (MeSH) terms will be used to search for eligible trials in the databases as mentioned above with no language restrictions.

Participant or population: Patients with heart, valve, or aortic disease are treated surgically under extracorporeal circulation and there will be no restrictions on sex, ethnicity, economic status, and education.

Intervention: Cardiac surgery with cardiopulmonary bypass with or without prophylactic corticosteroid administration. For comparator study arms, trials with concomitant study arms on other interventions were not excluded, as long as patients in the comparator arm received the same treatment as the corticosteroid arm except for corticosteroid administration.

Comparator: Cardiac surgery with cardiopulmonary bypass with or without prophylactic corticosteroid administration. For comparator study arms, trials with concomitant study arms on other interventions were not excluded, as long as patients in the comparator arm received the same treatment as the corticosteroid arm except for corticosteroid administration.

Study designs to be included: Randomized controlled trials (RCTs) of interest, published 0 . 
Eligibility criteria: Only randomized controlled clinical trials comparing corticosteroid with placebo or equal volume of normal saline, initiated either before or at the time of cardiopulmonary bypass were included. Studies that used unequal concurrent medical therapies or studies that evaluated corticosteroid in offpump cardiac surgery were excluded.

Information sources: Two reviewers (CTC, QZH) will search Pubmed, Web of Science, Embase, and ClinicalTrials, and Cochrane Central Register of Controlled Trials for relevant clinical trials published before August 1, 2020 without any language restrictions.

Main outcome(s): Mortality; occurrence of atrial fibrillation; myocardial infarction; pulmonary complications; acute kidney injury; postoperative infection; postoperative insulin use; gastro-intestinal bleeding;.

Additional outcome(s): Re-thoracotomy; neurological complications; inotropic use; blood transfusion; mechanical ventilation time; re-intubation; length of ICU stay; CRP/IL-6/IL-8 concentrations at 24 hours after cardiopulmonary bypass; vaso-active medication.

Data management: We will use the predesigned table to record the data extracted from the included trials. If relevant data of the trials is lost or unclear, we will consult the author via email before determining whether the study is included.

Quality assessment / Risk of bias analysis: The Cochrane Handbook for Systematic Reviews of Interventions will be used to assess the risk of bias of each trial included. The two authors (CTC, QZH) will evaluate the risk of bias based on the following domains: random sequence generation (selection bias), allocation concealment (selection bias), blinding of participants and personnel (performance bias), blinding of outcome assessment (detection bias), incomplete outcome data (attrition bias), selective outcome reporting (reporting bias), and other bias. The risk of bias in each domain will be assessed as as high, low, or uncertain and the results of the evaluation will be shown on the risk of bias graph.

Strategy of data synthesis: We will use Review Manager and Stata software to synthesise the data extracted. If the data extracted from the included studies are evaluated as highly homogeneous, we will conduct meta-analysis on them for the purpose of obtaining a clinically meaningful result. In order to carry out a standard meta-analysis, we will use the Chi2 and $\mathrm{I2}$ statistic test to evaluate statistical heterogeneity among the studies. If there is high heterogeneity (p50\%), we will use the DerSimonian and Laird random effect model to analyze the extracted data. Otherwise, we will adopt fixed-effect model to analyze the data. We will adopt the Mantel-Haenszel method to pool the binary data and the results will be reported in the form of relative risk (RR) with the 95\% confidence interval $(\mathrm{Cl})$. Inverse variance analysis method will be used to pool the continuous data and the results will be reported in the form of standardized mean difference (SMD) with 95\% confidence interval (Cl).

Subgroup analysis: If there is substantial heterogeneity and the available data are sufficient, we will perform subgroup analysis for searching potential origins of heterogeneity. If sufficient data are available, the included trials will be divided into four subgroups: $\leq 20 \mathrm{mg} / \mathrm{kg}$ (low dose), 20-40 $\mathrm{mg} / \mathrm{kg}$ (slightly high dose), 40-100 $\mathrm{mg} / \mathrm{kg}$ (high dose), and >100 $\mathrm{mg} / \mathrm{kg}$ (ultra high dose) based on the equivalent hydrocortisone dose.

Sensibility analysis: We will conduct sensitivity analysis to evaluate the robustness and the reliability of aggregation results by eliminating trials with high bias risk. If reporting bias exists, we will use the methods of fill and trim to analyze publication bias. 3.7. Publication bias Funnel charts and Egger's test will be adopted to assess publication bias if there are no less than ten eligible trials. If reporting bias is suspected in a trial, we 
will contact the corresponding author via email to find out whether there are additional outcome data which were not reported.

\section{Language: Without any language restrictions.}

Country(ies) involved: China.

Keywords: Cardiac surgery, cardiopulmonary bypass, steroid, inflammation, Meta-analysis.

Dissemination plans: The results of the study will be published in a peer-reviewed journal.

Contributions of each author:

Author 1 - Jian He - drafted the manuscript. Author 2 - Yuling Zhang - provided statistical expertise.

Author 3 - Zhihuang Qiu - contributed to the development of the selection criteria, and the risk of bias assessment strategy.

Author 4 - Tianci Chai - provided feedback and approved the final manuscript.

Author 5 - Guanhua Fang - provided feedback and approved the final manuscript.

Author 6 - Yunnan $\mathrm{Hu}$ - contributed to the development of the selection criteria, and the risk of bias assessment strategy.

Author 7 - Fan Xu - contributed to the development of the selection criteria, and the risk of bias assessment strategy.

Author 8 - Qiuyu Huang - contributed to the development of the selection criteria, and the risk of bias assessment strategy.

Author 9 - Hui Zheng - contributed to the development of the selection criteria, and the risk of bias assessment strategy.

Author 10 - Hao Zhou - contributed to the development of the selection criteria, and the risk of bias assessment strategy.

Author 11 - Mengyue Tian - contributed to the development of the selection criteria, and the risk of bias assessment strategy.

Author 12 - Liang Wan Chen - provided feedback and approved the final manuscript. 